\title{
Tunable synthesis of platinum nanoparticles by EtOH reduction in presence of poly (vinylpyrrolidone)
}

\author{
M. S. Rahman', S. Akhter ${ }^{2}$, K. N. Ahmed ${ }^{1}$, M. S. Rahman ${ }^{2}$, R. K. Saha ${ }^{2}$ and M. J. Hossain ${ }^{1,2 *}$ \\ ${ }^{1}$ Industrial Botany Research Division, BCSIR Laboratories Chittagong, Chittagong 4220, Bangladesh. \\ ${ }^{2}$ Forest Chemistry Division, Bangladesh Forest Research Institute, Chittagong 4211, Bangladesh.
}

\begin{abstract}
Monodispersed platinum nanoparticles (Pt-NPs) with an average diameter $d_{\mathrm{TEM}} 3.1 \pm 1.0 \mathrm{~nm}$ were synthesized by EtOH reduction method in the presence of an organic polymer poly (vinylpyrrolidone) (PVP) with an average molecular weight of 40,000. Using this particles as seed, size tunable Pt-NPs of $d_{\text {TEM }} 3.1 \pm 1.0 \mathrm{~nm}$ to $5.7 \pm 1.6 \mathrm{~nm}$ with sufficient monodispersity were synthesized by multiple step seeding growth. Formation of Pt-NPs was confirmed by the UV-visible absorption spectra. Transmission electron micrographs (TEM) and powder X-ray diffraction (XRD) patterns confirmed that the particles were single crystalline with fcc crystal geometry.
\end{abstract}

Key words: Platinum; EtOH; Nanoparticles; PVP; Synthesis

\section{Introduction}

Colloidal noble metal particles have recently attracted considerable attention in many areas of research due to their novel physical and chemical properties. Research on nanoscience has been growing steadily due to their unique position as a bridge between atoms and bulk solids and are of fundamental interest to both homogeneous and heterogeneous catalytic applications (Boudart, 1985; McLeod and Gladden, 1998). The electron confinement effect of nanocrystal is responsible for manipulating the electronic, optical, and magnetic properties of solid materials. Both catalytic activity (Rioux et al., 2005) and selectivity (Eppler et al, 2000) are known to be influenced by the size and the shape of the nanoparticles (Narayanan and El-Sayed, 2004; Tsung et al. 2009; Kuhn et al., 2008) and therefore the synthesis of well-controlled sizes and shapes of colloidal particles could be critical for this purpose.

Due to very tiny size, the size controlled synthesis is a big challenge for nano-research. Metal NPs can be prepared by physical and chemical methods. The physical methods like vapour deposition in principle subdivide bulk precursor to NPs. Chemical procedures involve reduction of metal ions to metal atoms, followed by controlled aggregation of atoms. Utilization of simple laboratory equipments and easy procedure to obtain small and uniform metal NPs made chemical reduction method unparallel for NPs synthesis. Pt-NPs are prepared in general, via the chemical reduction of
Pt ions by borohydride (Van Rheenen et al., 1987; Knecht et al., 2008), hydrogen (Ahmadi et al. 1996), alcohols (Toshima et al., 1991; Teranishi et al., 1999), glycol or ethylene glycol (Herricks et al., 2004) in the presence of a stabilizer or on a solid support. Despite a large choice of synthetic protocols, an accurate control of the particle size, simple and economical strategies for synthetic method is most important to investigate their novel physical and chemical properties.

Typically nanoparticles provide highly active centers which are not in a thermodynamically stable state due to their high surface energies (Schmid et al., 1996; Doyle et al., 2003). Soluble organic polymers are widely employed supports for synthesizing metal NPs because of their availability and enhanced stabilization properties (Toshima et al., 1991). PVP is the most studied polymer that can not only control the size of the particles but also dictates the shape as well. PVP molecules interact strongly through their carbonyl group with the Pt surface for their enhance stabilization (Teranishi et al., 1999). In the typical mechanism of PVP protected metal nanoparticles formation involve three steps: (I) coordination of PVP and metal ions, (II) PVP-promoted nucleation that produces smaller particles, and (III) stabilization of formed particles through physical and chemical bonding with PVP by steric shielding and also minimizing the particles surface energies which inhibited particle-particle contact and, thus, the agglomeration of particles.

*Corresponding author: E-mail: smjakir080@yahoo.com 
Chemical syntheses offer a versatile route allowing tailoring of the properties of materials by assembling atoms and particles from the atomic or molecular state to the macroscopic scale (Elechiguerra et al., 2006). The characteristics of the crystals can be controlled by the thermodynamics and kinetics of the synthesis (Goia, 1998) and the great progress has been made. However, there is still need for development of chemical methods that can tailor the morphology of Pt crystals at different scales.

This article describes a simple preparation and size control of small Pt-NPs stabilized by PVP with narrow size distribution by $\mathrm{EtOH}$ reduction method. The nanoparticles were characterized by UV-Visible optical spectroscopy, transmission electron microscopy (TEM) and powder x-ray diffractometry (XRD).

\section{Materials and methods}

\section{Chemicals and materials}

Hydrogen hexachloroplatinate(IV) hexahydrate $\left(\mathrm{H}_{2} \mathrm{PtCl}_{6} \cdot 6 \mathrm{H}_{2} \mathrm{O}\right)$ (Sigma-Aldrich), ethanol $\left(\mathrm{C}_{2} \mathrm{H}_{6} \mathrm{O}\right)$ (Merck), Poly(vinylpyrrolidone) (PVP, MW 40,000) (TCI), were of analytical grade and used as received. Double distilled water was used to prepare aqueous solutions. All of the glasswares along with teflon coated magnetic stirrer were cleaned with aqua regia for fine cleaning followed by rinsing with double distilled water.

\section{Preparation of Pt:PVP NPs}

As reported earlier (Hossain et al., 2012, Teranishi et al., 1999), a mixture of ethanol/water solution (9:1, v/v, $100 \mathrm{~mL}$; $90 \% \mathrm{EtOH})$ containing $\mathrm{H}_{2} \mathrm{PtCl}_{6}(30 \mathrm{mM}, 3.34 \mathrm{~mL})$ and $\mathrm{PVP}$ (444 mg, PVP-monomer/ $\mathrm{Pt}=40: 1$ ) was refluxed at $90^{\circ} \mathrm{C}$ in a $150 \mathrm{~mL}$ round bottom flask for $3 \mathrm{~h}$ using water bath. After cooling to room temperature, the produced Pt:PVP was purified using a dialysis membrane of cut off molecular weight $10 \mathrm{kDa}$ for the removal of inorganic ions and ethanol. Finally, the purified sample was stored in an air tight desiccators after freeze drying.

\section{Larger Pt:PVP by seed mediated growth}

To form larger Pt-NPs, smaller Pt:PVP NPs were used as seed particles. In a typical procedure, a mixture of total 25 $\mathrm{mL}$ aqueous solution of $\mathrm{H}_{2} \mathrm{PtCl}_{6}(10 \mathrm{mM}, 2.5 \mathrm{~mL})$ and ethanol/water solution $(9: 1, \mathrm{v} / \mathrm{v}, 22.5 \mathrm{~mL})$ were added to the $25 \mathrm{~mL}$ of Pt:PVP seed solution of $1.0 \mathrm{mM}$ concentration. The final concentration of $\mathrm{Pt}$ in the resultant $50 \mathrm{~mL}$ mixed solution was $1.0 \mathrm{mM}$ including both seeds and ions $(0.5 \mathrm{mM}$ from precursor solution and $0.5 \mathrm{mM}$ from seed particles).
PVP concentration was adjusted to PVP-monomer to Pt-atom ratio 40 by addition of $222 \mathrm{mg}$ extra PVP to the solution before refluxing. To reduce, the mixed solution was refluxed at $90{ }^{\circ} \mathrm{C}$ in a $100 \mathrm{~mL}$ round bottom flask for $3 \mathrm{~h}$ in air using water-bath. Initially prepared Pt:PVP NPs sample (step-1) was used as seed particles for larger Pt:PVP sample (Step-2). Similarly, step-2 Pt:PVP sample was used as seed for step-3 particles and step-3 sample was used as seed for step-4 particles. The as prepared larger Pt:PVP samples were purified by removing the inorganic ions and ethanol. Finally, the samples were stored in an air tight desiccators after freeze drying.

\section{Purification and storage of Pt:PVP samples}

The Pt:PVP organosols were diluted with equal volume of deionized water. This diluted organosols were centrifuged through a dialysis membrane of cutoff molecular weight (MWCO) $10 \mathrm{kDa}$ (Sartorius Stedim Biotech) with the help of a centrifuge machine (International Equipment Company, Boston Mass, USA) at $4000 \mathrm{rpm}$. When the volume was decreased to $\sim 2 \mathrm{~mL}$, it was redispersed with deionized water to $20 \mathrm{~mL}$ and recentrifuged to decrease the volume to $\sim 2 \mathrm{~mL}$. This process was repeated for 3 times. Finally, the purified Pt:PVP hydrosols were stored in an air tight desiccator after freeze drying.

\section{Characterization}

\section{$U V$-visible spectroscopy}

UV-visible spectra measurements (Shimadzu UV-1800 spectrophotometer) of colloidal solutions were carried out at BCSIR Laboratories, Chittagong equipped with a $1 \mathrm{~cm}$ path length quartz cuvette at room temperature.

\section{Transmission electron microscopy (TEM)}

Electron micrographs were obtained using a JEM-2100F transmission electron microscopy (TEM) instrument at Catalysis Research Center, Hokkaido University, Japan operated at accelerating voltage of $200 \mathrm{kV}$. The samples were prepared followed by drop-casting and air drying of diluted Pt:PVP solution on a carbon coated TEM grid. From the representative TEM micrographs, the diameters of more than $300 \mathrm{Pt}-\mathrm{NPs}$ were measured randomly. Their distributions were calculated from the histograms of the nanoparticles diameters using Gaussian function.

\section{$X$-ray diffractometry (XRD)}

X-ray diffraction (XRD) measurements (Bruker D8 Advance diffractometer) were performed at Catalysis Research Center, 
Hokkaido University, Japan. The XRD machine was operated at $40 \mathrm{kV}$ and $40 \mathrm{~mA}$ using a $\mathrm{Cu}-\mathrm{K}_{\alpha}(1.5418 \AA)$ radiation. The average diameters of the Pt crystallites $\left(d_{\mathrm{XRD}}\right)$ were calculated using the Scherrer formula to the $\mathrm{Pt}(111)$ diffraction peak. The XRD diffraction patterns were simulated using TOPAS-3 program.

$$
d_{x \times o}=\frac{k \lambda}{\beta \cos \theta} \text { Scherrer equation }
$$

Where $\mathrm{k}$ is a dimensionless constant called shape factor (for spherical particles its value is equal to 0.89$), \lambda$ is the $\mathrm{X}$-ray wavelength in nanometer ( $\mathrm{nm}), \theta$ is the Bragg diffraction angle of the $\operatorname{Pt}(111)$ plane, and $\beta$ is the full width at half-maximum (FWHM) of the Pt (111) diffraction peak.

\section{Results and discussion}

Purification is very important for successful characterization of NPs. Excess solvents, salts or unreduced ions were incorporated with NPs during synthesis. Followed by preparation, Pt:PVP was purified by using a $10 \mathrm{kDa}$ MWCO membrane dialyzer that permitted all impurities through it pores. The average MW of PVP (40 kDa) molecules were much larger than the MWCO of the membrane used $(10 \mathrm{kDa})$ and produced Pt-NPs were strongly coordinated with PVP (Hossain et al., 2012; Borodko et al., 2007; Tsunoyama et al., 2009) hence, neither PVP molecules nor Pt-NPs were identified in the ultrafiltrates which suggested that only impurities passed out through the purification process.

Due to the partial negative charge transfer effect of PVP to the metal ions, PVP molecules formed a coordinated complex with $\mathrm{Pt}^{4+}$ ions $[\mathrm{Pt}: \mathrm{PVP}]^{4+}$ which is the first step to the synthesis of Pt:PVP NPs (Hossain et al., 2012; Borodko et al., 2007; Tsunoyama et al., 2009). This stronger coordination effect was reflected from the slightly expanded ligand field splitting of $\mathrm{Pt}_{5 \mathrm{~d}}$ orbital than the ligand field splitting for complexing with $\mathrm{Cl}^{-}$ions (Teranishi et al., 1999). As a result, the optical absorption spectra of $\mathrm{H}_{2} \mathrm{PtCl}_{6}$ solution containing PVP in EtOH was red shifted from $260.0 \mathrm{~nm}$ to $261.9 \mathrm{~nm}$ as shown in Fig.1.

The pale yellow color of the initial $\mathrm{H}_{2} \mathrm{PtCl}_{6}$ solution turned into dark brown within $30 \mathrm{~min}$ refluxing at $90{ }^{\circ} \mathrm{C}$ temperature, indicating complete reduction of all $\mathrm{Pt}^{4+}$ ions present in the solution:

$\mathrm{H}_{2} \mathrm{PtCl}_{6}+\mathrm{CH}_{3} \mathrm{CH}_{2} \mathrm{OH} \rightarrow \mathrm{Pt}^{0}+2 \mathrm{CH}_{3} \mathrm{CHO}+6 \mathrm{HCl}$

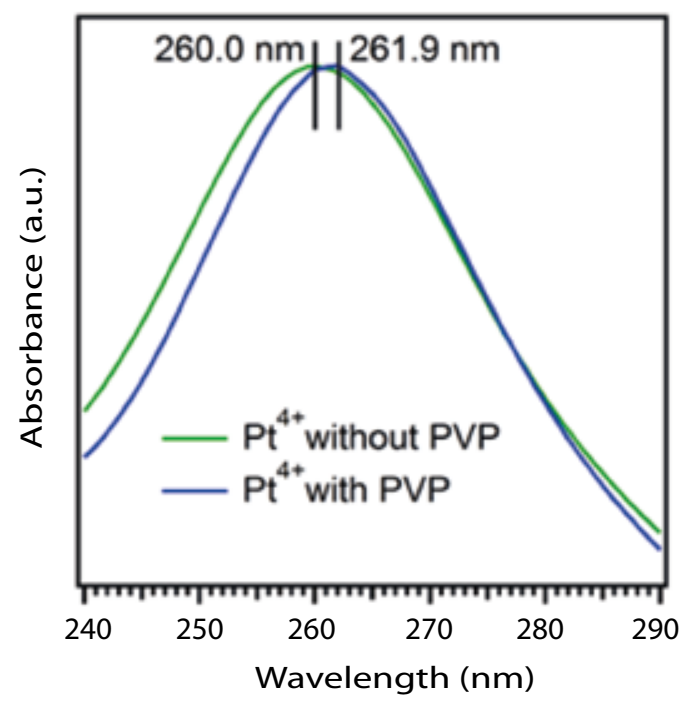

Fig. 1. UV-visible optical spectra of $\mathrm{H}_{2} \mathrm{PtCl}_{6}$ in $\mathrm{EtOH}$ containing PVP

Here the solution showing an absorption peak at $261.9 \mathrm{~nm}$ in UV-visible absorption spectrum gradually decreased and finally disappeared. The exhibition of exponential feature in the optical spectra suggested that the formation of band structure as well as the production of Pt-NPs (Rioux et al., 2005; Knecht et al., 2008; Toshima et al., 1991; Teranishi et al., 1999; Herricks et al., 2004; Hossain et al., 2012). Although the Pt nuclei began to form only after 20 min reflux and completed in $30 \mathrm{~min}$, there was no aggregation of NPs even after $3 \mathrm{~h}$ reflux at $90{ }^{\circ} \mathrm{C}$ temperature which suggested the robustness of Pt:PVP NPs formed (Fig. 2).

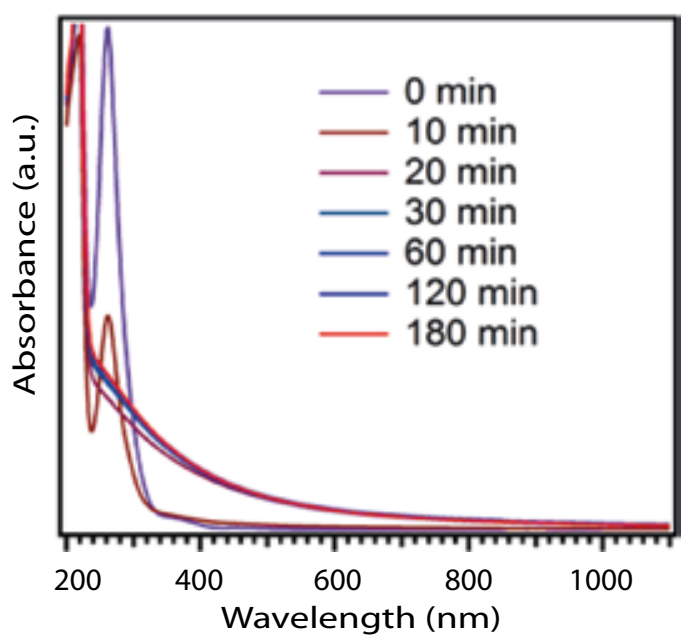

Fig. 2. UV-visible optical spectra of Pt:PVP sample prepared by EtOH reduction. In 30 min reflux, all the Pt precursor metal ions were formed to Pt-NPs. Even after 180 min reflux there was no change of spectra or color of the solution, i.e, particles 

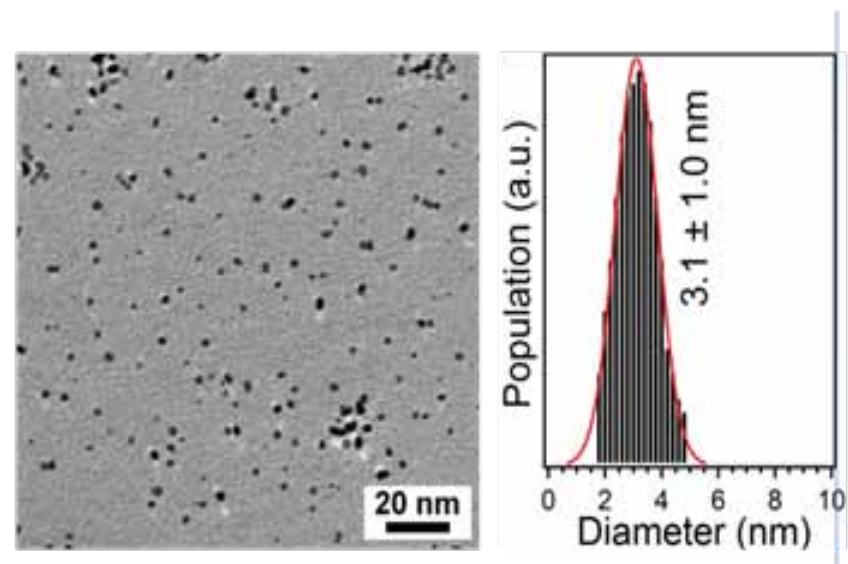

\section{Fig. 3. Shows the representative TEM image of Pt:PVP NPs by EtOH reduction}

TEM micrograph (Fig.3) shows that most of the NPs are comprised of spherical particles and those were of single crystalline. The diameter of more than 300 Pt-NPs were determined randomly and the average diameter is found to be $3.1 \pm 1.0 \mathrm{~nm}$.

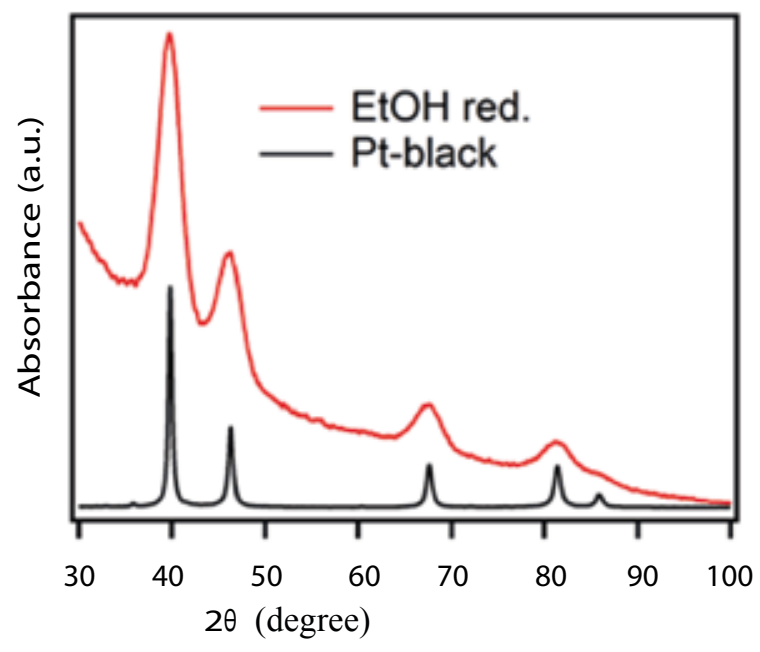

Fig. 4. Shows the powder XRD patterns of Pt:PVP along with bulk Pt

X-ray diffraction technique has been widely used in nanoparticle research as a primary characterization tool for obtaining some critical features such as crystal structure, crystallite size, strain and purity of the sample. Among other methods, the simplest and most widely used method for estimating the average crystallite size is from the full width at half maximum (FWHM) of a diffraction peak using the Scherrer equation as shown in characterization section. Fig.4
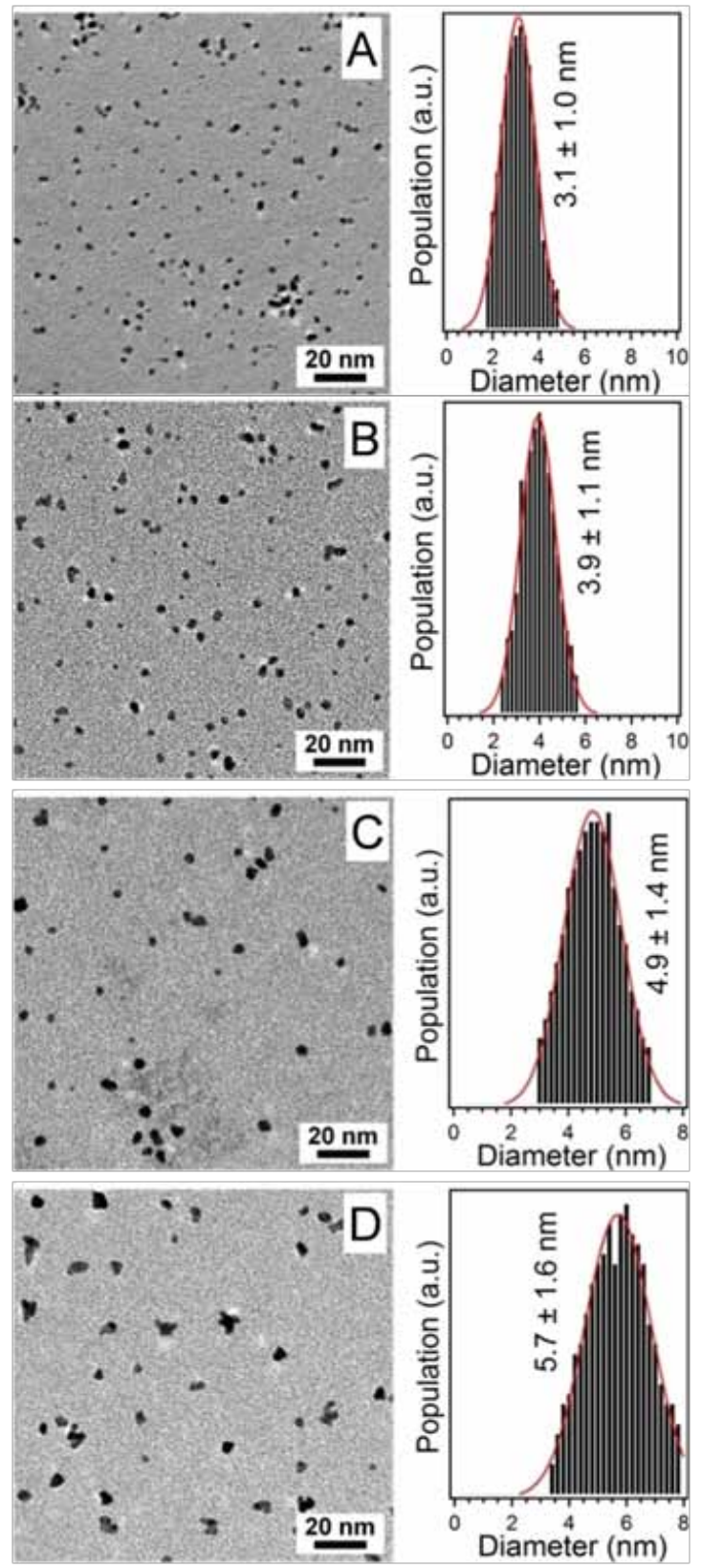

Fig. 5. (A) Repesentative TEM image for $1^{\text {st }}$ step Pt-NPs. (B) Repesentative TEM image for $2^{\text {nd }}$ step Pt-NPs. (C) Repesentative TEM image for $3^{\text {rd }}$ step Pt-NPs. (D) Repesentative TEM image for $4^{\text {th }}$ step Pt-NPs

illustrates the XRD pattern of the Pt-NPs. Using the Scherrer method and assuming the Gaussian profile for peak model, the average crystallite size was obtained as $3.1 \mathrm{~nm}$. As can be seen, these values are very close to the TEM value. The crystallite size determined by XRD analysis $\left(d_{\mathrm{XRD}}\right)$ was comparable with the core size distributions of Pt:PVP 
determined by TEM analysis. The XRD pattern indicated the formation of fcc crystallites of the particles which were same as bulk Pt.

The size of Pt:PVP particles is greatly influenced by the type and concentration of alcohol used as reducing agent. By single step synthesis, it is quite difficult to synthesize Pt:PVP NPs which are larger than $3.3 \mathrm{~nm}$ (Teranishi et al., 1999). To obtain larger NPs, the monodispersed single step synthesized Pt:PVP were used as seed particles for the stepwise growth to obtain nanoparticles larger than $3.1 \mathrm{~nm}$.

For the second growth of Pt:PVP NPs, a $100 \mathrm{~mL} 90 \%$ ethanolic solution containing seed particles (single step Pt:PVP NPs, $3.1 \mathrm{~nm}$ ) and $\mathrm{H}_{2} \mathrm{PtCl}_{6}$ were refluxed for $3 \mathrm{~h}$ in air. The total concentration of $\mathrm{Pt}$ was $1.0 \mathrm{mM}$ where seed particles concentration was $0.5 \mathrm{mM}$ and added $\mathrm{H}_{2} \mathrm{PtCl}_{6}$ concentration was $0.5 \mathrm{mM}$. PVP concentration was adjusted to PVP-monomer/Pt-atom $=40$ by addition of $222 \mathrm{mg}$ extra PVP to that solution before refluxing. Third and fourth growth of Pt:PVP NPs were conducted in a similar manner where second step growth particles were used as seed for third step growth particles and third step growth particles were used as seed for fourth step growth Pt:PVP NPs respectively.

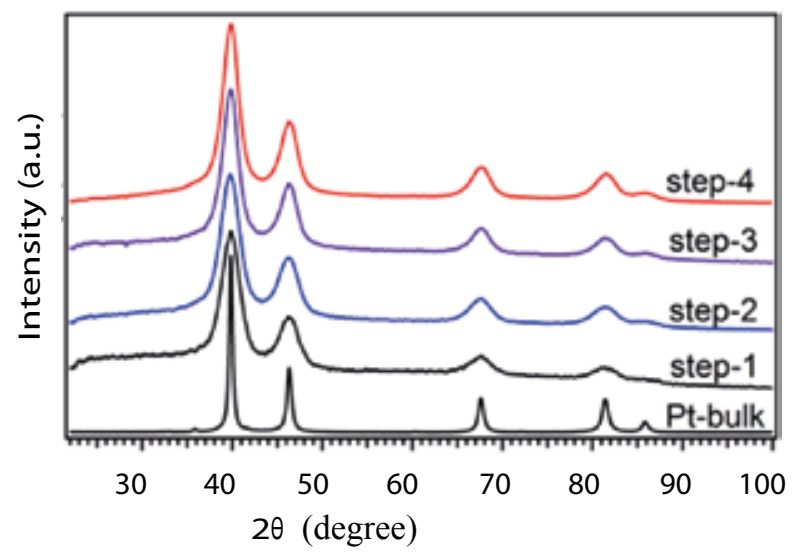

Fig. 6. Shows the powder XRD patterns of formed Pt:PVP along with bulk Pt

The representative TEM micrographs and size distributions of Pt:PVP obtained at each growth steps are shown in Fig.5. With increasing growth steps, the diameter of Pt:PVP NPs were clearly increased, where seed particles were served as nuclei for larger particles. The mean diameters of Pt:PVP determined by TEM measurements are $3.1 \pm 1.0,3.9 \pm 1.1$, $4.9 \pm 1.4$ and $5.7 \pm 1.6 \mathrm{~nm}$ for first, second, third and fourth steps growths respectively. The increment of their crystal sizes with broader distribution is clearly depicted in Fig.7 with each increment step. The size distribution of seed particles were also greatly influenced that of the stepwise-synthesized particles which are in good agreement with the literature (Teranishi et al., 1999). The crystallite size determined by XRD analysis $\left(d_{\mathrm{XRD}}\right)$ were $3.1,3.8,4.8$ and 5.6 $\mathrm{nm}$ for step-1, step-2, step-3 and step-4 respectively. The crystallite sizes determined by XRD are comparable with the core size distributions of Pt:PVP determined by TEM analysis as shown in Fig.6. The powder XRD pattern indicated the formation of fcc crystallites of produced particles those were same as bulk Pt.

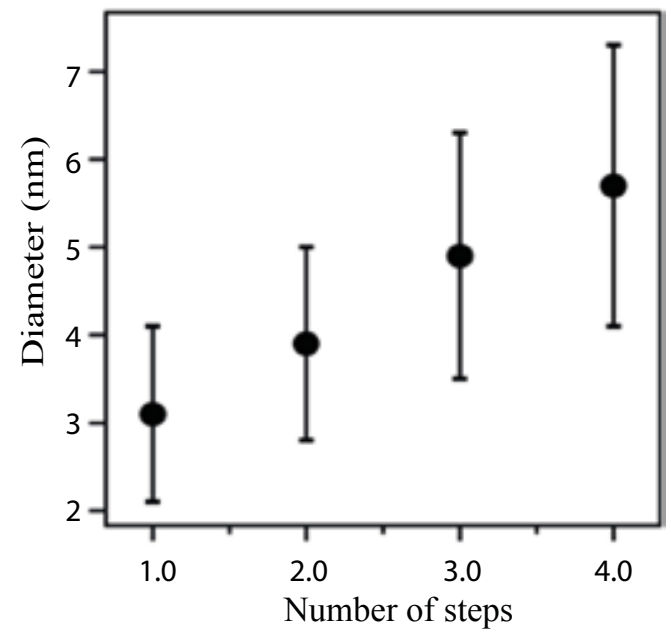

Fig. 7. Size and size distribution profile of Pt-NPs sythesized by multiple step growth

\section{Conclusion}

EtOH reduction is a simple and effective method for the production of small and monodispersed Pt:PVP NPs. Refluxing of the precursor $\mathrm{H}_{2} \mathrm{PtCl}_{6}$ solution with $90 \%$ ethanol at $90{ }^{\circ} \mathrm{C}$ temperature in air yielded $d_{\text {TEM }} 3.1 \pm 1.0 \mathrm{~nm}$ Pt-NPs. Stepwise seeded growth method, ethanolic particles were tuned from $3.1 \pm 1.0 \mathrm{~nm}$ to $5.7 \pm 1.6 \mathrm{~nm}$ with sufficient monodispersity. The crystallite sizes determined by XRD are comparable with the core size distributions of Pt:PVP determined by TEM analysis. Most of the particles are comprised of single crystal with spherical shape except seeded growth largest particles. They have fcc crystal geometry same as that of bulk platinum.

\section{Acknowledgement}

This research work was done under the fellowship programme of BCSIR. Thanks to BFRI authority for their kind cooperation. Finally special thanks to Catalysis Research Center, Hokkaido University, Japan for allowing us to take TEM images and measurements of XRD patterns and analysis. 


\section{References}

Ahmadi TS, Wang ZL, Green TC, Henglein A, El-Sayed MA (1996), Shape-controlled synthesis of colloidal platinum nanoparticles, Science. 272: 1924-1926.

Borodko Y, Humphrey SM, Tilley TD, Frei H, Somorjai GA (2007), Charge-transfer interaction of poly (vinylpyrrolidone) with platinum and rhodium nanoparticles, The Journal of Physical Chemistry $C$. 111: 6288-6295.

Boudart M (1985), Heterogeneous catalysis by metals, Journal of Molecular Catalysis. 30: 27-38.

Doyle AM, Shaikhutdinov SK, Jackson SD, Freund HJ (2003), Hydrogenation on metal surfaces: Why are nanoparticles more active than single crystals?, Angewandte Chemie International Edition. 42: 5240-5243.

Elechiguerra JL, Reyes-Gasga J, Yacaman MJ (2006), The role of twinning in shape evolution of anisotropic noble metal nanostructures, Journal of Materials Chemistry. 16: 3906-3919.

Eppler AS, Zhu J, Anderson EA, Somorjai GA (2000), Model catalysts fabricated by electron beam lithography: AFM and TPD surface studies and hydrogenation/dehydrogenation of cyclohexene $+\mathrm{H}_{2}$ on a Pt nanoparticle array supported by silica, Topics in Catalysis. 13: 33-41.

Goia D (1998), Preparation of monodispersed metal particles, New Journal of Chemistry. 22: 1203-1215.

Herricks T, Chen J, Xia Y (2004), Polyol synthesis of platinum nanoparticles: control of morphology with sodium nitrate, Nano Letters. 4: 2367-2371.

Hossain MJ, Tsunoyama H, Yamauchi M, Ichikuni N, Tsukuda T (2012), High-yield synthesis of PVP-stabilized small Pt clusters by microfluidic method, Catalysis Today. 183: 101-107.

Knecht MR, Weir MG, Myers VS, Pyrz WD, Ye H, Petkov V, Buttrey DJ, Frenkel AI, Crooks RM (2008), Synthesis and characterization of $\mathrm{Pt}$ dendrimer-encapsulated nanoparticles: effect of the template on nanoparticle formation, Chemistry of Materials. 20: 5218-5228.

Kuhn JN, Huang W, Tsung CK, Zhang Y, Somorjai GA (2008), Structure sensitivity of carbon- nitrogen ring opening: impact of platinum particle size from below 1 to $5 \mathrm{~nm}$ upon pyrrole hydrogenation product selectivity over monodisperse platinum nanoparticles loaded onto mesoporous silica, Journal of the American Chemical Society. 130: 14026-14027.

Narayanan R, El-Sayed MA (2004), Shape-dependent catalytic activity of platinum nanoparticles in colloidal solution, Nano Letters. 4: 1343-1348.

McLeod A, Gladden L (1998), Relating metal particle geometry to the selectivity and activity of supported-metal catalysts: A Monte Carlo study, Journal of Catalysis. 173: 43-52.

Rioux RM, Song H, Hoefelmeyer JD, Yang P, Somorjai GA (2005), High-surface-area catalyst design: synthesis, characterization, and reaction studies of platinum nanoparticles in mesoporous SBA-15 silica, The Journal of Physical Chemistry B. 109: 2192-2202.

Schmid G, Maihack V, Lantermann F, Peschel S (1996), Ligand-stabilized metal clusters and colloids: Properties and applications,. Journal of the Chemical Society, Dalton Transactions. 5: 589-595.

Teranishi T, Hosoe M, Tanaka T, Miyake M (1999), Size control of monodispersed Pt nanoparticles and their 2D organization by electrophoretic deposition, The Journal of Physical Chemistry B. 103: 3818-3827.

Toshima N, Harada M, Yonezawa T, Kushihashi K, Asakura K (1991), Structural analysis of polymer-protected palladium/platinum bimetallic clusters as dispersed catalysts by using extended x-ray absorption fine structure spectroscopy, The Journal of Physical Chemistry. 95: 7448-7453.

Tsunoyama H, Ichikuni N, Sakurai H, Tsukuda T (2009), Effect of electronic structures of Au clusters stabilized by poly (N-vinyl-2-pyrrolidone) on aerobic oxidation catalysis, Journal of the American Chemical Society. 131: 7086-7093.

Tsung CK, Kuhn JN, Huang W, Aliaga C, Hung LI, Somorjai GA, Yang P (2009), Sub-10 nm platinum nanocrystals with size and shape control: catalytic study for ethylene and pyrrole hydrogenation, Journal of the American Chemical Society. 131: 5816-5822.

Van Rheenen PR, McKelvy MJ, Glaunsinger WS (1987), Synthesis and characterization of small platinum particles formed by the chemical reduction of chloroplatinic acid, Journal of Solid State Chemistry. 67: 151-169.

Received: 25 January 2015; Revised: 05 April 2015; Accepted: 30 June 2015. 